\title{
Brief exposure to a novel stimulus during imprinting in chicks and its influence on subsequent preferences
}

\author{
PATRICK BATESON \\ Sub-Department of Animal Behaviour, University of Cambridge \\ Madingley, Cambridge CB3 $8 A A$, England
}

\begin{abstract}
In the course of imprinting, chicks were regularly tested by brief exposure to a novel stimulus in successive choice tests. Their approach towards the novel stimulus relative to their approach towards the familiar was used as a measure of preference. The repeated tests with the novel stimulus were started at four different times after the beginning of imprinting: $21,43,65$, and $87 \mathrm{~min}$. The first test of each group showed the longer that chicks had been imprinted beforehand, the greater the preference for the familiar. However, chicks trained for $21 \mathrm{~min}$ before testing were influenced by the first test and continued to show a preference for the more novel stimulus throughout subsequent testing. By contrast, the other three groups showed a stable preference for the familiar stimulus.
\end{abstract}

When day-old chicks that have been isolated from hatching are exposed to a visually conspicuous object, they will commonly approach it and behave towards it as they would towards their natural mother. The effect of the exposure is to narrow a chick's preference to the familiar object so that subsequently the bird will prefer that object to other visually conspicuous objects that would have been just as attractive if the chick had been first exposed to one of them instead. The phenomenon by which social preferences are narrowed to a particular object is commonly known as "imprinting," but it has become apparent that the narrowing is not an all-ornothing process as the term implies (e.g., Zajonc et al., 1973). Moreover, my own evidence suggests that the learning curve is not monotonic (Bateson, 1973a; Bateson \& Jaeckel, 1976; Jackson \& Bateson, 1974).

Generally, the time course of the emerging preference for the familiar object has been studied by comparing the behavior in a choice test of independent groups of birds exposed to an object for different lengths of time. The reason for doing this rather than testing the same birds many times is simple. A choice test usually takes a substantial proportion of the total time for which a chick has been exposed to visually conspicuous objects, and so subsequent tests may be contaminated by experience with the novel object in the first test. In this paper, I describe an attempt to shorten the time of each

This work was supported by a grant from the Science Research Council. I am particularly indebted to Tom Goss for making the apparatus, which was beautiful as well as reliable. I also thank Priscilla Barrett for her help in running the experiments. choice test, in the hope that a repeatedly tested bird might behave like one that was trained for the same length of time but not previously tested. Although the attempt was not wholly successful, the procedure proved to have a number of other advantages. It allowed chicks to be tested in the apparatus in which they were exposed and in which their behavior was automatically recorded. This greatly reduced the handling time and also meant that many birds could be trained and tested simultaneously. Furthermore, the experiment confirmed that chicks' preferences for the familiar object develop in a gradual and somewhat unexpected way.

\section{METHOD}

\begin{abstract}
Subjects
Thirty-two domestic chicks drawn equally from two batches were used. Fertile eggs of a broiler strain (Ross Chunkies from Hall Farm, Herringswell, Suffolk) were placed in an automatic incubator for 17-18 days, after which they were transferred to a dark still-air incubator where they were hatched. The chicks were transferred, generally with $3 \mathrm{~h}$ of hatching, to another dark incubator and kept there in individual compartments until the beginning of the experiment. Handling of the chicks was kept to a minimum and was always done in the dark or with the aid of a dim green light, to which chicks are relatively unresponsive (Kovach, 1971).
\end{abstract}

\section{Apparatus}

The apparatus for training and testing the chicks was modified from that described in an earlier paper (Bateson \& Jaeckel, 1974). It consisted of eight running wheels, and one chick was placed inside each wheel. Through the curved mesh of the wheel on which a chick walked it could see a flashing light. The bottom of the light was $8 \mathrm{~cm}$ above the bottom of the wheel and $40 \mathrm{~cm}$ away from it. The light, which was different from that used in previous experiments, was a $2-\mathrm{cm}$ slit moving upwards at a maximum rate of $47 \mathrm{~cm} / \mathrm{sec}$ in a window $10 \mathrm{~cm}$ wide $\times 15 \mathrm{~cm}$ deep. The rate 
was such that the stimulus presented four separate upward moving slits per second: this flash rate has been found optimally attractive to day-old chicks (Gottlieb \& Simner, 1969; Simner, 1973). The light was produced by a $12-\mathrm{V}, 45-\mathrm{W}$ bulb. Each stimulus was equipped with two bulbs; one was covered by a Cinemoid Primary Red filter and the other by a Primary Yellow filter, but only one bulb was lit at any one time. The bulbs were surrounded by a drum $(15 \times 15 \mathrm{~cm}$ diam) fitted with translucent slits and driven on a horizontal axis by an electric synchronous motor at $60 \mathrm{rpm}$.

Above the flashing light was a loudspeaker which emitted the natural maternal call of the domestic hen at an average intensity of $65 \mathrm{~dB}$ (re: $20 \mu \mathrm{N} / \mathrm{m}^{2}$ ). This was measured from the chick's position with a Dawe sound-level meter (Type 1400G, A scale) using the fastest response; the peak intensity was $68 \mathrm{~dB}$. The maternal call phrase was repeated every $3.6 \mathrm{sec}$ and consisted of seven segments.

A pair of wheels were placed in front of each flashing light and loudspeaker. Each wheel was set at about $20^{\circ}$ from a horizontal line drawn at right angles to the front of the stimuli. Chicks placed in each wheel could see and hear the stimuli clearly. They could not see each other but they could hear the calls of the other chick. Two wheels and a set of stimuli were housed within a temperature-controlled cabinet (Bateson \& Jaeckel, 1974). The inside of the cabinet was painted matt black and lit by an overhead $240-\mathrm{V}, 25-\mathrm{W}$ bulb placed midway between the centers of the two wheels. The temperature was kept between $29.8^{\circ}$ and $30.2^{\circ} \mathrm{C}$.

The flashing light, the maternal call, and the overhead light in each of four cabinets were all turned on and off simultaneously by a timer (see the Procedure section for the schedule). Each chick's approach activity and withdrawal activity were recorded separately by means of a magnet on the side of each wheel which closed one of two reed switches according to which direction the chick was moving (see Bateson \& Wainwright, 1972, for details). The reed switches were connected to a computer-based event recorder (White, 1971). In this experiment, a chick obtained only one approach count as it drove the wheel through a complete revolution; in previous experiments, chicks obtained four counts.

\section{Procedure}

All chicks were exposed separately to static patterned white light for $30 \mathrm{~min}$ immediately prior to training (see Bateson \& Wainwright, 1972).

Training with either a red or a yellow flashing light began 16-22 $\mathrm{h}$ after hatching. Half the chicks were trained with the red light and half with the yellow one. Eight chicks at a time were exposed to the stimuli, which were turned on and off automatically, according to the following schedule: $9 \mathrm{~min}$ on, $1 \mathrm{~min}$ off, $1 \mathrm{~min}$ on, $1 \mathrm{~min}$ off, $1 \mathrm{~min}$ on, $1 \mathrm{~min}$ off, $9 \mathrm{~min}$ on ... and so on, until the chicks had been in the wheels for $112 \mathrm{~min}$ and exposed to the stimuli for $88 \mathrm{~min}$. After $21 \mathrm{~min}$ of training with one light, one red-trained and one yellow-trained chick were exposed for $1 \mathrm{~min}$ to the color they had not seen before. In other words, they received a short period of exposure to a novel visual stimulus in the second of the 1 -min periods of exposure that followed a 9-min period. After $43 \mathrm{~min}$ of training with one flashing light, two more chicks received their first test with a novel color and, similarly, two other groups received their first test after 65 and $87 \mathrm{~min}$, respectively. After the first exposure to a novel stimulus, each chick was exposed again to that stimulus following $10 \mathrm{~min}$ of further training with the more familiar visual stimulus. After $88 \mathrm{~min}$ of exposure to flashing lights, all eight chicks were withdrawn from the wheels and placed in a dark incubator. Meanwhile, another eight chicks were treated in the same way as the first lot had been. About $2 \mathrm{~h}$ after the end of their first period of exposure to the stimuli, each chick was returned to a wheel and given a further $44 \mathrm{~min}$ of exposure. This time, all chicks received $1 \mathrm{~min}$ exposure to the less familiar visual stimulus after $10 \mathrm{~min}$ of
Table 1

The Number of Chicks in Each Group and the Number of Tests With a Novel Stimulus Given During Training

\begin{tabular}{|c|c|c|c|c|}
\hline & \multicolumn{4}{|c|}{$\begin{array}{l}\text { Length of Training } \\
\text { in Minutes Before } \\
\text { First Test }\end{array}$} \\
\hline & 21 & 43 & 65 & 87 \\
\hline Number of chicks & 8 & 8 & 8 & 8 \\
\hline Tests in first part of training & 7 & 5 & 3 & 1 \\
\hline Tests in second part of training & 4 & 4 & 4 & 4 \\
\hline
\end{tabular}

exposure to the more familiar one, which was presented on the same schedule as in the first part of the experiment. The number of chicks in each group and the number of tests with the less familiar visual stimulus received by each group in each part of training are shown in Table 1.

\section{RESULTS}

Changes in the chicks' attempts to approach the more familiar visual stimuli are shown in Figure 1. The results are very similar in pattern to those of Bateson and Jaeckel (1974). (The difference between the experiments in absolute approach counts arose simply because in the present experiment one revolution of a wheel generated only one count, whereas in the previous experiment, it generated four counts.) The gap shown in Figure 1 between the eighth and ninth 9-min training sessions represents the period of nearly $2 \mathrm{~h}$ that the chicks spent in a dark incubator. Clearly some "warm-up" occurs after such a rest, although it is not so marked as the initial increase in approach activity shown by naive birds. The variation was such that none of the groups differed significantly from any of the other groups in approach activity.

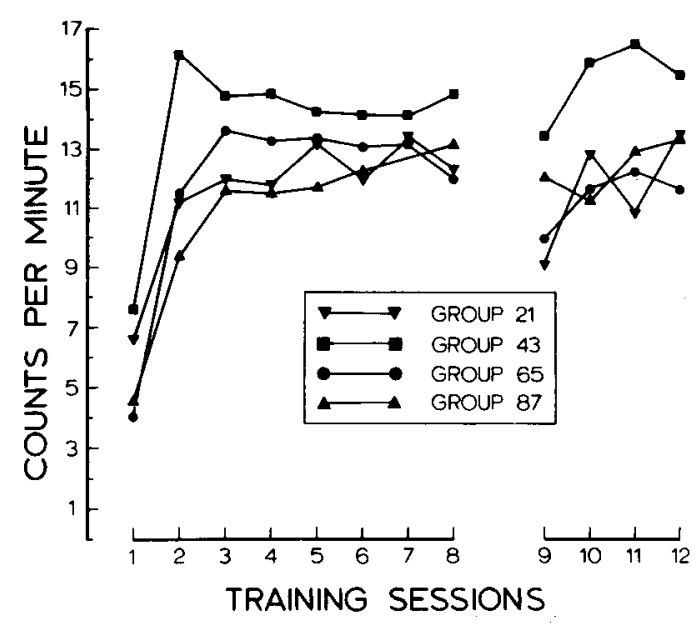

Figure 1. Mean approach counts per minute in each 9-min training session. Ten approach counts is approximately equivalent to a forward movement of $10 \mathrm{~m}$. The four groups, which received their first test 21, 43, 65, or 87 min after the beginning of training, did not differ during training. 


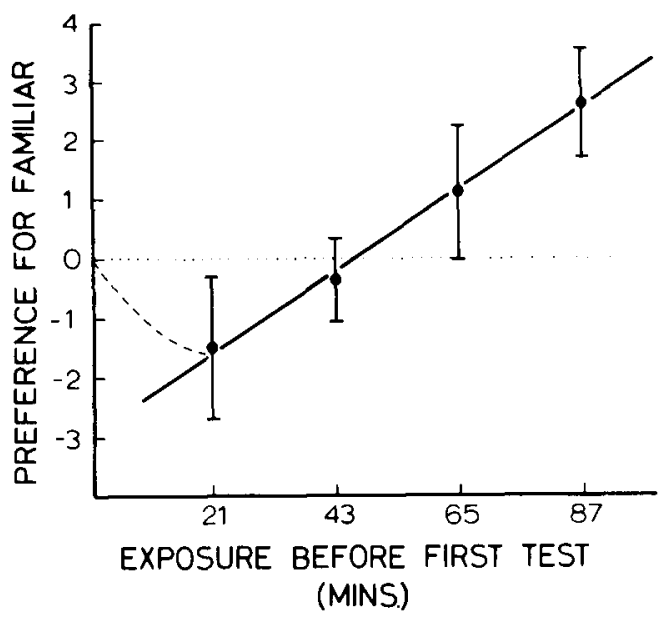

Figure 2. Preferences for the familiar measured in the first successive choice test after different amounts of exposure. Means and standard errors are given. Preference for the familiar is the difference between approach counts towards the familiar stimulus and approach counts towards the novel stimulus. Each group consisted of eight chicks. The equation for the regression line fitted to the data is: $y=.063 x-2.94$.

The results of the first successive choice test given to each group are shown in Figure 2. What is shown is the absolute difference between the counts while approaching the familiar and the counts while approaching the novel one. The slope of the regression line fitted to all the data shown in Figure 1 is significantly greater than zero $[\mathrm{t}(30)=3.18, \mathrm{p}<.01]$. When the difference between approach to the familiar and approach to the novel are averaged over all tests, a different picture emerges (Table 2). The mean preference for the novel in the group given its first test after $21 \mathrm{~min}$ stabilizes and is marginally below the chance level [matched pairs $t(7)=2.01$, $p<.05$, one-tailed]. The mean preferences for the familiar of the other three groups are indistinguishable, and when the results are lumped, are significantly above the chance level [matched pairs, $t(23)$ $=2.27, p<.05]$. The difference between mean preference for Group 21 and the overall mean for the other three groups is highly significant $[\mathrm{t}(30)=$ $2.85, \mathrm{p}<.01]$.

\section{DISCUSSION}

The results show that even a relatively brief pulse of training with a novel stimulus can influence a chick's subsequent response to that stimulus. The chicks in the group given the first test after $21 \mathrm{~min}$ of training continued to show a slight preference for the novel object in subsequent tests despite prolonged experience with the more familiar stimulus. Their behavior did not come to resemble that of the chicks given their first tests after longer periods of training. In other words, the behavior of chicks in subsequent choice tests can be contaminated by their experience of the first test. This means that repeated testing of the same chicks is not a reliable way of describing the subtle changes in preference taking place in the early stages of imprinting.

The experiment demonstrated the possibility of measuring the outcome of imprinting by one successive choice test. The advantage of the procedure is that the birds do not have to be removed from the apparatus in which they have been trained before being tested. This means that they are handled much less than in studies in which the birds have to be transferred to special test apparatus (e.g., Bateson \& Jaeckel, 1974; Bateson \& Wainright, 1972). Furthermore, since the birds are placed in running-wheels, their approach (and withdrawal) when confronted with a particular stimulus is easily recorded automatically, both during training and testing. On the face of it, a successive choice is likely to be a lesssensitive test of preference than a simultaneous choice. Even so, the first successive choice tests given to each group in the present study showed clearly how preference for the familiar is a function of the length of exposure (see Zajonc et al., 1973). Furthermore, the technique picked up once again the tendency of chicks to prefer slight novelty when exposed to it at a sufficiently early stage of imprinting (see Bateson, 1973a; Bateson \& Jaeckel, 1976; Jackson \& Bateson, 1974).

Why should the birds ever show a preference for an object which they have not been exposed to over one they have seen before? Their behavior did not arise because the novel stimulus was intrinsically more attractive, since the experimental design was balanced and half the chicks were exposed to one stimulus and half to the other. Nor does their interest in novelty appear to stem from the unnatural stimuli which were used, since I have found, in unpublished experiments, that chicks will work for novel views of a rocking stuffed hen. Furthermore, when given a choice between the rocking hen and the red flashing light used in the present study, naive chicks showed a slight preference for the light. At a functional level,

Table 2

Mean Preference for the Familiar Stimulus in All Choice Tests

\begin{tabular}{crc}
\hline & \multicolumn{2}{c}{ Preference } \\
\cline { 2 - 3 } Bength of Exposure & Mean & SE \\
\hline 21 & -1.62 & .80 \\
43 & 1.32 & .61 \\
65 & .52 & .68 \\
87 & 1.37 & 1.18 \\
\hline
\end{tabular}

Note-Preference for the familiar is the difference between approach counts toward the familiar stimulus and approach counts toward the novel stimulus. A difference of one count is approximately equal to travelling $1 \mathrm{~m}$ more toward one stimulus in 1 min than the other. Each group consisted of eight chicks. 
the preference for slight novelty may reflect a selection pressure during evolution to respond quickly to the mother regardless of whether she is seen from the front or back (Bateson, 1973a); a young bird that rejected all except the first view it had of its mother would probably be at a disadvantage compared with another that developed preferences for all commonly seen views of its mother. What the chick detects as being optimally discrepant from the familiar may objectively resemble the familiar object more and more closely the longer the bird has been trained with one object (Bateson, 1973a, 1973b). Such a process would enable the bird to optimize between restricting its visual preferences to a view of its mother with which it already had had visual experience and remaining receptive to the possibility that she might look different when viewed from another side.

\section{REFERENCES}

Bateson. P. P. G. Internal influences on early learning birds. In R. A. Hinde \& J. Stevenson Hinde (Eds.), Constraints on learning. London: Academic Press, 1973. Pp. 101-116. (a)

Bateson, P. P. G. Preference for familiarity and novelty: A model for the simultaneous development of both. Journal of Theoretical Biology, 1973, 41, 249-259. (b)
Bateson. P. P. G., \& JAECkel, J. B. Imprinting: Correlations between activities of chicks during training and testing. Animal Behaviour, 1974, 22, 899-906.

Bateson, P. P. G., \& Jaeckel, J. B. Chicks' preference for familiar and novel conspicuous objects after different periods of exposure. Animal Behaviour, 1976, 24, 386-390.

Bateson, P. P. G., \& Wainwright, A. A. P. Effects of prior exposure to light on the imprinting process in domestic chicks. Behaviour, 1972, 42, 279-290.

Gortlieb, G., \& Simner, M. L. Auditory versus visual flicker in directing the approach response of domestic chicks. Journal of Comparative and Physiological Psychology, 1969, 67, 58-63.

JaCkson, P. S., \& Bateson, P. P. G. Imprinting and exploration of slight novelty in chicks. Nature, 1974, 251, 609-610.

Kovach, J. K. Effectiveness of different colours in the elicitation and development of approach behaviour in chicks. Behaviour. 1971, 38. 154-168.

Simner, M. L. The development of visual flicker rate preference in the newly hatched chick. Developmental Psychobiology, 1973, 6. 377-384.

WHTE, R. E. C. WRATS: A computer-compatible system for automatically recording and transcribing behavioural data. Behaviour, 1971, 40, 135-161.

ZAJONC, R. B., REIMER, D. J., \& HAUSSER, D. Imprinting and the development of object preference in chicks by mere repeated exposure. Journal of Comparative and Physiological Psychology, $1973,83,434-440$.

(Received for publication December 7, 1977; revision accepted July 14,1978 .) 